\title{
Very Late Stent Thrombosis 89 Months after Implantation of Durable Polymer Everolimus-Eluting Stent due to Late Acquired Stent Malapposition and Uncovered Struts Observed by Optical Coherence Tomography, Near-Infrared Spectroscopy and Coronary Angioscopy
}

\author{
Takuya Tsujimura, MD, Takayuki Ishihara, MD, Shodai Kawanami, MD, Osamu Iida, MD, \\ Mitsutoshi Asai, MD, PhD, Masaharu Masuda, MD, PhD, Shin Okamoto, MD, \\ Kiyonori Nanto, MD, Takashi Kanda, MD, Shota Okuno, MD, \\ Yasuhiro Matsuda, MD, Yosuke Hata, MD, and Toshiaki Mano, MD, PhD \\ Kansai Rosai Hospital Cardiovascular Center, Amagasaki, Hyogo, Japan
}

\begin{abstract}
Very late stent thrombosis (VLST) is a potentially life-threatening complication in coronary angioplasty patients. The patient was a 95-year-old man who had been implanted with a durable polymer everolimus-eluting stent (DP-EES, $3.5 \times 23 \mathrm{~mm}$ ) at the restenosis site in the proximal part of the left descending artery using only plain balloon angioplasty for acute coronary syndrome (ACS) 27 months earlier. Final intravascular ultrasound (IVUS) evaluation had shown no stent malapposition and adequate stent expansion. However, he presented to our hospital due to vomiting and fatigue 89 months after the implantation. An electrocardiogram showed ST-elevation in V2-6 leads, we therefore suspected ACS and emergent coronary angiography (CAG) revealed total occlusion of DP-EES site, which was diagnosed as VLST. Percutaneous coronary intervention (PCI) was subsequently carried out and complete recovery of blood flow was obtained by thrombus aspiration. Optical coherence tomography (OCT) demonstrated uncovered struts, stent malapposition, and some thrombi in the proximal part of DP-EES. Yellow chemogram was not detected in the DP-EES site by near-infrared spectroscopy (NIRS). Coronary angioscopy (CAS) showed exposed stent struts with red thrombus adhesion in the proximal part of the DP-EES. Judging from intravascular images, the main cause of VLST was stent malapposition and uncovered struts. Here, we report a case with VLST due to late acquired stent malapposition and uncovered struts 89 months after DP-EES implantation.
\end{abstract}

Key words: very late stent thrombosis, percutaneous coronary intervention, durable polymer drugeluting stent, late acquired stent malapposition

\section{Introduction}

Very late stent thrombosis (VLST) is a rare but potentially life-threatening complication after stent implantation. The incidence of VLST after second-generation drug-eluting stent (DES) implantation has decreased compared with that

\footnotetext{
Kansai Rosai Hospital Cardiovascular Center, 3-1-69 Inabaso, Amagasaki, Hyogo 660-8511, Japan

E-mail: t.tsujimura0110@gmail.com

(Received: 2019.11.28; Accepted:2020.5.15)

doi: 10.15791/angioscopy.cr.20.0031
}

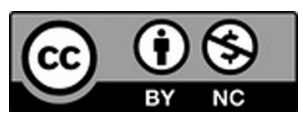

This work is licensed under a Creative Commons [Attribution-NonCommercial 4.0 International] License.

(C) 2020 Japanese Association of Cardioangioscopy of first-generation DES implantation. ${ }^{1)}$ However, VLST remains a major concern in the clinical setting. In this report, we present a case with VLST 89 months after implantation of durable polymer everolimus-eluting stent (DP-EES) due to late acquired stent malapposition and uncovered struts observed by optical coherence tomography (OCT), nearinfrared spectroscopy (NIRS), and angioscopy.

\section{Case Presentation}

A 95-year-old man was transferred to our hospital due to vomiting and fatigue. He had a medical history of hypertension and paroxysmal atrial fibrillation (PAF) with an episode of acute myocardial infarction (AMI) approximately 116 months earlier and had undergone percutaneous coronary intervention (PCI) for total occlusion of the proximal part of the left anterior descending artery (LAD) with 
collaterals from the left circumflex artery (Fig. 1A). After balloon dilation, the lesion had not been sufficiently dilated due to a severely calcified plaque (Fig. 1B). Therefore, the procedure had been terminated without stent implantation because blood flow was deemed acceptable and there was a concern about stent underexpansion if a stent was implanted. Follow-up coronary angiography (CAG) 27 months after the initial PCI had demonstrated severe stenoses at the proximal and middle part of the LAD (Fig. 1C). Therefore, he underwent an additional PCI for these lesions. Because of severe calcification of the lesion, rotational atherectomy with a $1.5-\mathrm{mm}$ burr and a $1.75-\mathrm{mm}$ burr had been performed. After performing rotational atherectomy, one DP-EES (Xience $^{\mathrm{TM}}, 2.5 \times 15 \mathrm{~mm}$, Abbott Vascular, Abbott Park, IL, USA) had been implanted in the middle part of LAD and another DP-EES (PROMUS ${ }^{\mathrm{TM}}$, $3.5 \times 23$ mm, Boston Scientific Co., Marlborough, MA, USA) had been implanted in the proximal part of LAD. Finally, excellent blood flow had been achieved demonstrated by angiography (Fig. 1D). We judged that intravascular ultrasound
(IVUS) evaluation had shown no major stent malapposition with adequate stent expansion, although we did not perform contrast IVUS, coronary angioscopy (CAS), or OCT at that time (Fig. 2A-E). After the second PCI, he was free from angina. Although the duration of dual antiplatelet therapy was unknown, it was switched to single anticoagulant therapy using edoxaban (30 mg/day) which was appropriate dose for this patient.

On this admission (present report), although the abdominal computed tomography findings had led to the diagnosis of acute cholecystitis by a gastroenterologist, he was transferred to cardiovascular center because the electrocardiogram on admission showed ST-elevation in V2-6 leads. Acute coronary syndrome (ACS) was suspected, and emergent $\mathrm{CAG}$ was conducted. Emergent $\mathrm{CAG}$ revealed total occlusion of the DP-EES site in the proximal part of LAD, which was diagnosed as VLST (Fig. 1E). PCI was subsequently carried out and complete recovery of blood flow was achieved after thrombus aspiration (Fig. 1F). On pathological evaluation, the aspirated matter was composed of

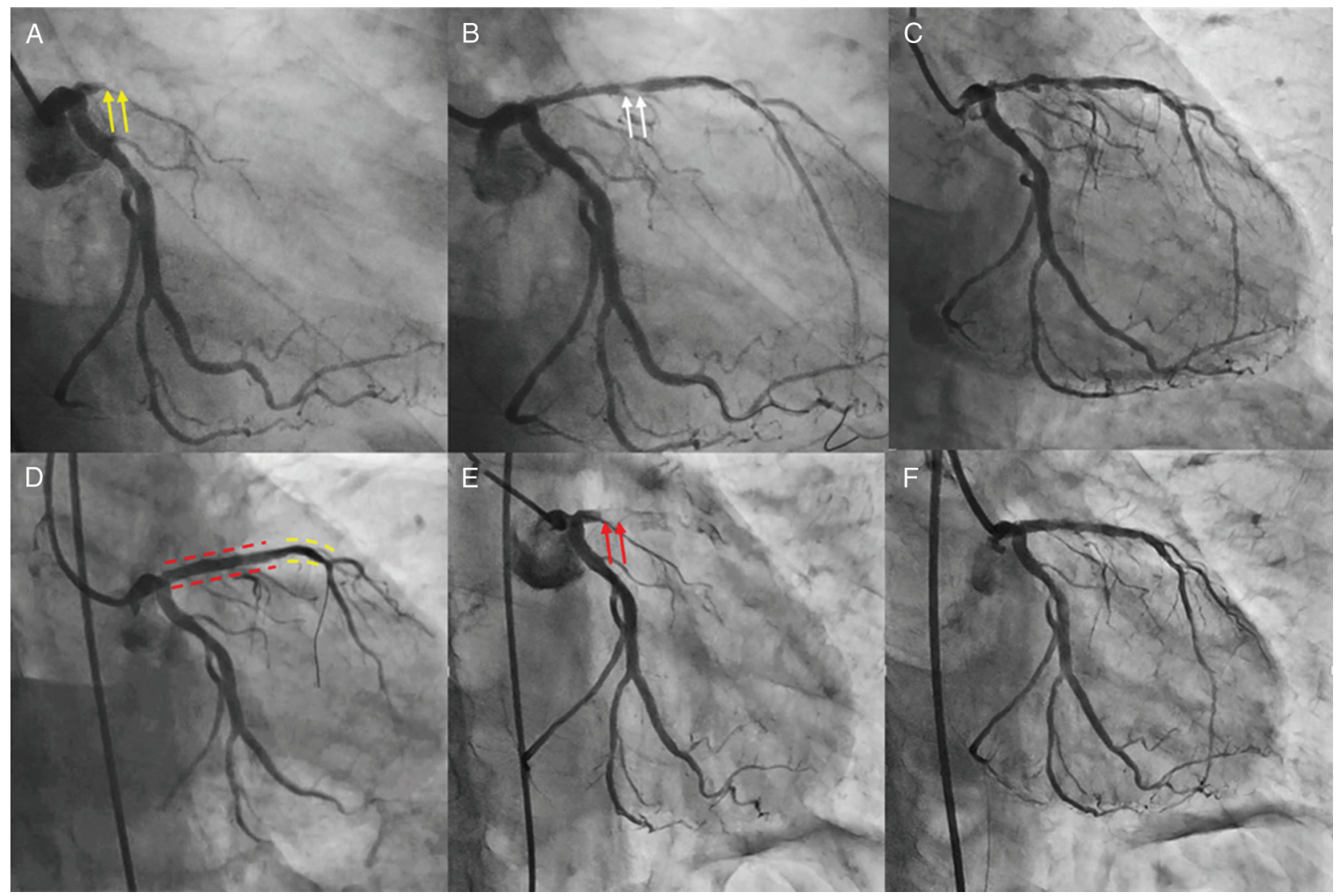

Fig. 1 Images of CAG. (A) Initial CAG. CAG revealed total occlusion of the proximal part of the LAD (yellow arrows). (B) CAG immediately after initial PCI. After balloon dilation, the lesion was not sufficiently dilated due to a severely calcified plaque (white arrows). (C) Follow-up CAG 27 months after initial PCI. Follow-up CAG revealed severe stenoses at the proximal and middle part of the LAD. (D) Additional PCI 27 months after initial PCI. DP-EES was implanted in the middle part of LAD and one more DP-EES was implanted in the proximal part of LAD. Dashed red line: DP-EES implantation site; Dashed yellow line: Second DP-EES implantation site. (E) CAG 89 months after additional PCI. Very late stent thrombosis occurred 89 months after DP-EES implantation (red arrows). (F) Final CAG after PCI. After thrombus aspiration, complete recovery of blood flow was achieved. CAG: coronary angiography; DP-EES: durable polymer everolimus-eluting stent; LAD: left anterior descending artery; PCI: percutaneous coronary intervention 


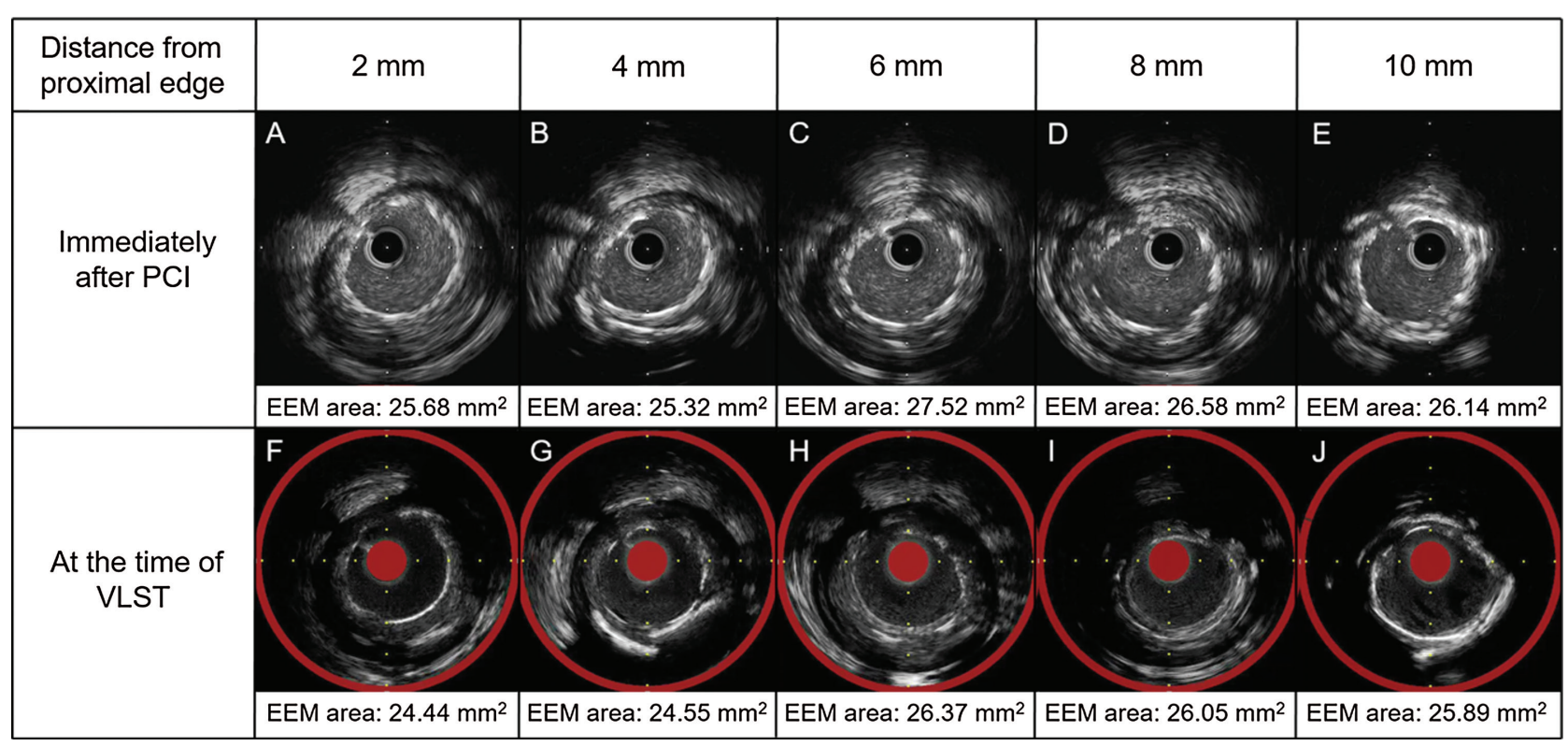

Fig. 2 Images of IVUS immediately after stent implantation and at the time of VLST. (A-E) Image of IVUS immediately after stent implantation. EEM areas were $25.68 \mathrm{~mm}^{2}, 25.32 \mathrm{~mm}^{2}, 27.52 \mathrm{~mm}^{2}, 26.58 \mathrm{~mm}^{2}$, and $26.14 \mathrm{~mm}^{2}$ at $2 \mathrm{~mm}, 4 \mathrm{~mm}, 6 \mathrm{~mm}, 8 \mathrm{~mm}$, and $10 \mathrm{~mm}$ distal to the proximal edge of the DP-EES, respectively. (F-J) Image of IVUS at the time of VLST. EEM area were $24.44 \mathrm{~mm}^{2}, 24.55 \mathrm{~mm}^{2}, 26.37 \mathrm{~mm}^{2}$, $26.05 \mathrm{~mm}^{2}$, and $25.89 \mathrm{~mm}^{2}$ at $2 \mathrm{~mm}, 4 \mathrm{~mm}, 6 \mathrm{~mm}, 8 \mathrm{~mm}$, and $10 \mathrm{~mm}$ distal sites to the proximal edge of the DP-EES, respectively. DP-EES: durable polymer everolimus-eluting stent; EEM: external elastic membrane; IVUS: intravascular ultrasound; VLST: very late stent thrombosis

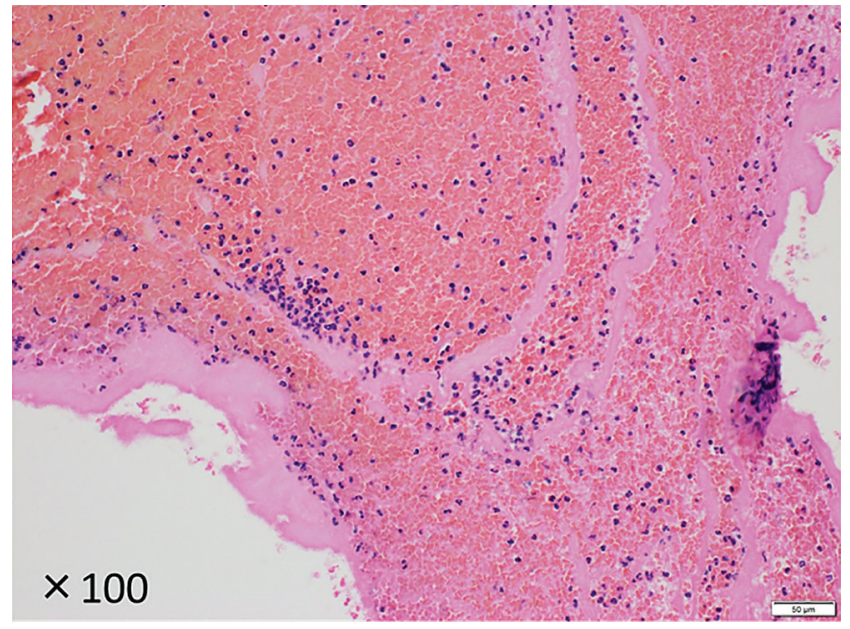

Fig. 3 Histopathology of aspirated materials. H \& E stain of the aspirated materials showing fresh mixed fibrin thrombi without plaque, eosinophils, or cholesterol cleft.

fresh platelet thrombi with fibrin and without plaque, eosinophil, or cholesterol cleft (Fig. 3). Then, we evaluated the DP-EES site in the proximal part of LAD using intravascular imaging devices. OCT demonstrated uncovered struts, stent malapposition, and some thrombi in the proximal part of DP-EES site (Figs. 4C and 4D), although OCT showed homogeneous neointima in the distal part of DP-EES site (Fig. 4E). Yellow chemogram which represents lipid was not detected by NIRS in the proximal and distal part of DP-EES site (Figs. 4F-H and 4L). CAS showed that stent struts were exposed with red thrombus adhesion in the proximal part of DP-EES site (Figs. 4I and 4J), while stent struts were embedded by the neointima in the distal part of DP- EES site (Fig. 4K). We completed the procedure because we acquired an excellent angiographic result after thrombus aspiration. After PCI, the peak values of creatine kinase (CK) and CK-MB were $4741 \mathrm{U} / \mathrm{L}$ and $400 \mathrm{U} / \mathrm{L}$, respectively. Low-density lipoprotein cholesterol level was $55 \mathrm{mg} / \mathrm{dL}$. Dual antiplatelet therapy with aspirin and prasugrel started in addition to anticoagulant therapy with edoxaban. Although he gradually recovered in the early days, his respiratory condition got worse drastically because of acute interstitial pneumonia 12 days after the hospitalization. Despite maximum support including steroid pulse therapy, the patient died due to respiratory failure 20 days after the hospitalization.

\section{Discussion}

Here, we reported a case with VLST occurring 89 months after implantation of DP-EES observed by OCT, NIRS, and CAS. Although there was a possibility of embolization due to PAF, the pathological evaluation of the aspirated thrombus demonstrated fresh platelet thrombus with fibrin, suggesting that the thrombus was organized in the coronary artery rather than thromboembolism.

Previous OCT reports showed that stent malapposition, neoatherosclerosis, and uncovered struts are the main causes of VLST. ${ }^{2-4)}$ In the present case, neoatherosclerosis could not be considered as the cause of VLST because yellow chemogram which represents lipid was not detected by NIRS although CAS showed light yellow plaque. Therefore, since 


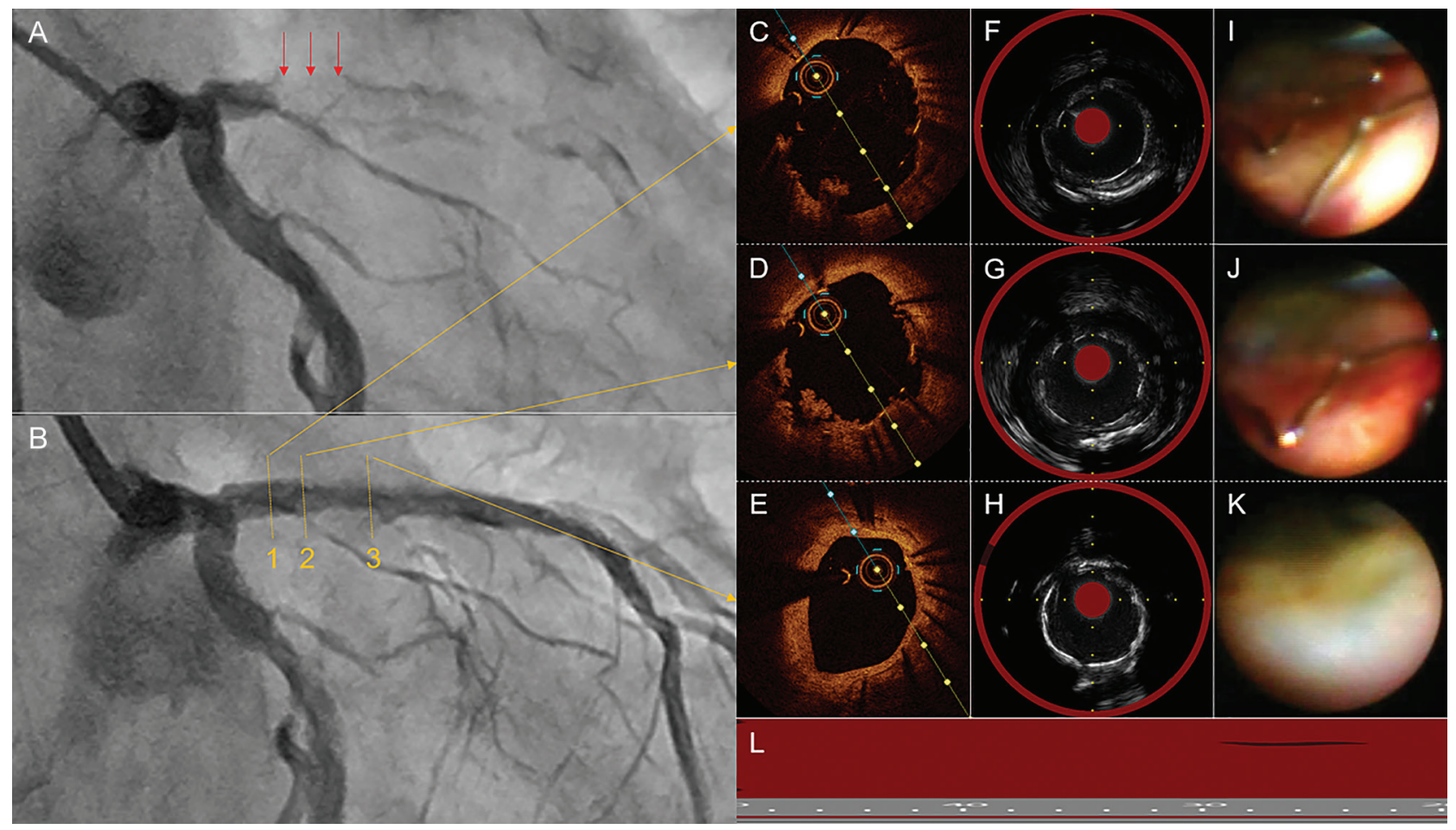

Fig. 4 Images of intravascular images. (A) CAG at the time of VLST. CAG demonstrated the total occlusion of DP-EES site (red arrows). (B) CAG after PCI for VLST. After thrombus aspiration, complete recovery of blood flow was obtained. (C-E) Images of OCT. OCT demonstrated exposed struts, stent malapposition, and some thrombi in the proximal part of DP-EES site (C and D), although OCT showed homogeneous neointima in the distal part of DP-EES site (E). (F-H) Images of NIRS-IVUS. NIRS-IVUS showed no yellow chemogram in DP-EES site (F, G, and H). (I-K) Images of CAS. CAS showed that stent struts were exposed with red thrombus adhesion in the proximal part of DP-EES site (I and J), while stent struts were embedded by the neointima in the distal part of DP-EES site (K). (L) Chemogram of NIRS. Yellow chemogram was not detected.

CAG: coronary angiography; CAS: coronary angioscopy; DP-EES: durable polymer everolimus-eluting stent; OCT: optical coherence tomography; NRIS-IVUS: near-infrared spectroscopy intravascular ultrasound; PCI: percutaneous coronary intervention; VLST: very late stent thrombosis

calcification is sometimes detected as yellow color by CAS, yellow plaque in Figs. 4I and 4J may be calcification. On the other hand, OCT demonstrated uncovered struts and stent malapposition and CAS showed the fully visible struts without neointima. Previous pathological report also mentioned that widely uncovered struts were one of the risk of stent thrombosis.5) Judging from the intravascular images, the major cause of VLST was thought to be related to stent malapposition and uncovered struts. Several mechanisms of stent malapposition have been proposed as follows: (1) positive vessel remodeling; (2) dissolution of jailed thrombus or plaque debris; and (3) stent underexpansion. ${ }^{6}$ In this case, stent underexpansion and stent malapposition were not observed by IVUS images immediately after stent implantation. Therefore, it was considered more likely that stent malapposition occurred in the chronic phase with late acquired malapposition. Positive vessel remodeling was not found by IVUS because external elastic membrane area of DP-EES implantation site did not change from immediately after stent implantation to the time of VLST occurrence (Fig. 2). Therefore, dissolution of jailed thrombus or plaque debris was a possible mechanism of late acquired stent malapposition in this case. The tissue under the DP-EES could have contained thrombus because the stent was implanted in the restenosis site after balloon dilatation of the lesion. In this case, it is possible that the thrombus under the stent dissolved in the chronic phase, which might have caused late acquired stent malapposition. To the best of our knowledge, this is the first report demonstrating VLST of DP-EES due to late acquired stent malapposition by the dissolution of thrombus or plaque debris.

A previous study reported that DES use for AMI patients presenting for coronary intervention was associated with delayed arterial healing compared with patients with stable angina. ${ }^{7)}$ In this case, arterial healing of the lesion with VLST might have been delayed and so the stent struts were uncovered because it was originally an AMI-related lesion. Regarding patient-related factors, inflammation and hypovolemia due to acute cholecystitis would promote thrombogenicity. In addition, even with the appropriate dose of edoxaban, single anticoagulant drug therapy without antiplatelet therapy may have contributed to the development of VLST. Therefore, it might be necessary to continue antiplatelet therapy in ACS-related lesions due to possibility of stent malapposition and uncovered struts because high thrombogenicity conditions would be a long-term risk. In 
addition, if stent malapposition is observed in the chronic phase, it may be necessary to perform additional balloon dilation to obtain adequate stent apposition.

\section{Disclosure Statement}

The authors have no conflict of interest to disclose.

\section{References}

1) Räber L, Magro M, Stefanini GG, et al: Very late coronary stent thrombosis of a newer-generation everolimus-eluting stent compared with early-generation drug-eluting stents: a prospective cohort study. Circulation 2012; 125: 1110-1121

2) Taniwaki M, Radu MD, Zaugg S, et al: Mechanisms of very late drug-eluting stent thrombosis assessed by optical coherence tomography. Circulation 2016; 133: 650-660

3) Lee SY, Ahn JM, Mintz GS, et al: Characteristics of earlier versus delayed presentation of very late drug-eluting stent thrombosis: an optical coherence tomographic study. J Am Heart Assoc 2017; 6: e005386.

4) Adriaenssens T, Joner M, Godschalk TC, et al: Optical coherence tomography findings in patients with coronary stent thrombosis: a report of the PRESTIGE Consortium (Prevention of late stent thrombosis by an interdisciplinary global european effort). Circulation 2017; 136: 1007-1021

5) Finn AV, Joner M, Nakazawa G, et al: Pathological correlates of late drug-eluting stent thrombosis: strut coverage as a marker of endothelialization. Circulation 2007; 115: 2435-2441

6) Cook S, Wenaweser P, Togni M, et al: Incomplete stent apposition and very late stent thrombosis after drug-eluting stent implantation. Circulation 2007; 115: 2426-2434

7) Nakazawa G, Finn AV, Joner M, et al: Delayed arterial healing and increased late stent thrombosis at culprit sites after drug-eluting stent placement for acute myocardial infarction patients: an autopsy study. Circulation 2008; 118: 1138-1145 